\title{
Study of Serum Leptin in Well-differentiated Thyroid Carcinoma: Correlation with Patient and Tumor Characteristics
}

\author{
Rania Abdel Rehem • Waleed Abo Elwafa • \\ Reham Abo Elwafa - Tarek Ezzat Abdel-Aziz
}

Published online: 28 May 2014

(c) The Author(s) 2014. This article is published with open access at Springerlink.com

\begin{abstract}
Background There is a proven relationship between obesity and several cancers including breast, endometrium, colorectal, and esophagus. With the increasing incidence of both obesity and thyroid cancer, we designed the present study to investigate a causal relationship between leptin, which is one of the well known adipokines, and well-differentiated thyroid cancer (WDTC).

Methods Serum leptin levels were measured in 30 patients with WDTC and compared to 30 healthy control subjects before and 1 month after surgery. Other parameters studied included age, sex, body mass index, menopausal status in women, lymph node status, tumor size, and disease multifocality.

Results There were no differences between the two groups regarding age and sex. Preoperative leptin levels were higher in the WDTC patients when compared to the control
\end{abstract}

\section{R. A. Rehem}

Internal Medicine Department, Endocrinology, Faculty of Medicine, Alexandria University, Alexandria, Egypt

W. A. Elwafa - T. E. Abdel-Aziz

Department of General Surgery, Endocrine Unit, Faculty of Medicine, Alexandria University, Alexandria, Egypt

\section{R. A. Elwafa}

Department of Clinical Pathology, Faculty of Medicine, Alexandria University, Alexandria, Egypt

T. E. Abdel-Aziz ( $\square)$

General and Endocrine Surgery Unit, University College London Hospital, 235 Euston Road, London NW1 2BU, UK

e-mail: t.abdel-aziz@ucl.ac.uk

T. E. Abdel-Aziz

Division of Surgery and Interventional Science, University

College London, London, UK patients [19.25 (1.50-109.60) vs $0.90(0.50-11.80) \mathrm{ng} / \mathrm{ml}$, $p<0.001$, group 1 vs group 2, respectively]. A significant drop in leptin levels 1 month after surgery occurred in the WDTC group, falling from $19.25(1.50-109.60)$ to 0.90 $(0.60-8.90) \mathrm{ng} / \mathrm{ml}(p<0.001)$. This did not occur in the control group $(p=0.274)$. Lymph node involvement, tumor size, and multifocality had no effect on leptin levels, although trends were observed $(p=0.48,0.079$, and 0.064), respectively.

Conclusions Serum leptin levels were significantly higher in WDTC patients when compared to control group patients, with a significant drop after surgery. Leptin may play a role in diagnosis of WDTC; however, its prognostic value is still undetermined.

\section{Introduction}

Obesity is a worldwide major health issue with an increasing prevalence [1]. In addition to being a medical condition increasing the risk of heart disease and diabetes, obesity is associated with several cancers through a family of metabolically active adipocytokines including tumor necrosis factor (TNF)-alpha, interleukin (IL)-6, type 1 plasminogen activator inhibitor, adiponectin, and leptin [25]. The exact mechanisms explaining this association are still at large; however, several theories have been proposed, including hyperinsulinemia and a state of chronic low level inflammation associated with obesity, with abnormal cytokine production affecting tumor initiation and progression [6,7]. Leptin, one of the most abundant and most investigated adipokines, was thought to have a role in regulating body weight solely by regulating body fat stores, but leptin has functions that extend beyond food intake and energy balance regulation, being also a metabolic and 
neuroendocrine hormone. Leptin has roles in glucose metabolism, normal sexual maturation, and reproduction, and it also has effects on the hypothalamic-pituitaryadrenal, thyroid and growth hormone axes, hematopoiesis, and the immune system [8,9]. With respect to its diverse functions in immunity, leptin may have a major role in oxidation, which is an important risk factor in carcinogenesis. Indeed, obesity has long been recognized to be a risk factor for tumorigenesis, and accumulating evidence suggests that leptin is a potential link between obesity and cancer development. Further studies have shown it to act as a mitogenic agent promoting the proliferation and invasiveness of certain cancer cells [10].

Papillary thyroid carcinoma (PTC) is the most common neoplasm of the thyroid, accounting for $80-85 \%$ of all thyroid cancers [11]. Increasing incidence of differentiated thyroid cancers has been observed in both men and women across all tumor sizes [12]. PTC is found in a variety of morphologic variants, like the follicular variant of papillary thyroid carcinoma (FVPTC). It is usually clinically indolent, although rarely it may present with local invasion or distant metastases, which may adversely affect survival [13]. The relation between obesity and thyroid cancer has recently been studied, showing a higher prevalence of thyroid cancer in the obese population [14]. The present study was designed to investigate the relation between serum levels of leptin and well-differentiated thyroid carcinoma (WDTC) and to evaluate its correlation with patient and tumor characteristics.

\section{Methods}

Sixty patients were enrolled in this study and were divided into two groups. Group $1(n=30)$ comprised patients with WDTC diagnosed by fine needle aspiration cytology (FNAC), and group $2(n=30)$ who were the control patients with benign thyroid nodules. All patients included in this study were admitted to Alexandria Main University hospital between September 2011 and September 2012. An informed consent form was signed by all patients according to a protocol approved by the Ethics Committee of the hospital.

All patients were subjected to complete physical examination, standard biochemical tests, and thyroid function tests. Both FNAC and ultrasound scans were performed in all patients, with a CT requested when necessary to evaluate extrathyroidal tumor invasion.

Exclusion criteria included diabetes mellitus, hypertension, thyroid hormonal profile abnormalities, either hyperthyroidism or hypothyroidism, and ongoing hormonal therapy, such as oral contraceptive pills, estrogen preparations, and thyroid hormone preparations. Patients with evidence of recent systemic illness or receiving medications for other medical conditions were also excluded from the study (Fig. 1).

All patients underwent either total thyroidectomy (TT) or hemithyroidectomy. Central neck dissection (CND) was done for those with positive central lymph nodes, and functional neck dissection (FND) was performed in patients with lateral nodal neck disease. Histopathological examination of the resected specimens was performed. Pathological criteria including tumor size, tumor pathological type, extrathyroidal invasion, multifocality, lymphovascular invasion, lymph node status, and presence of lymphocytic thyroiditis were reported.

Patient demographics and anthropometric measurements including weight, height, and body mass index (BMI) were recorded. Samples were collected with the patient in the fasting state in the morning. Sample collection, processing, and storage were done according to the instructions of the reference laboratory and the kits. Samples for leptin level measurements were taken from the patients preoperatively and again 1 month postoperatively.

Assays

Serum samples were kept at $-40{ }^{\circ} \mathrm{C}$ until analysis. All samples from each patient were run in the same assay. Serum leptin was measured by the DIAsource LeptinEASIA Kit, (DIAsource ImmunoAssays, Louvain-la-Neuve, Belgium).

Statistical analysis

Data were collected, tabulated, and then analyzed using IBM SPSS software package version 20.0., and statistically analyzed with the Shapiro-Wilk test, the D'Agastino test, and the Mann-Whitney test. Significance of the obtained results was judged at the $5 \%$ level.

\section{Results}

In group 1, 15 (50\%) patients underwent TT with FND, 5 (16.7\%) patients underwent TT with CND, 8 (26.7\%) patients underwent TT, and $2(6.6 \%)$ patients who had a diagnosis of micropapillary carcinoma underwent hemithyroidectomy with no further surgery. In group 2 (controls) $22(73.3 \%)$ patients underwent TT and 8 (26.7 \%) patients underwent hemithyroidectomy. All patients had their leptin levels measured preoperatively and 1 month after the operation.

Demographics and patient characteristics

There was no significant difference between the two groups regarding age and gender. The BMI for all patients was 
Fig. 1 Flow chart showing the patients with thyroid disease excluded from the study

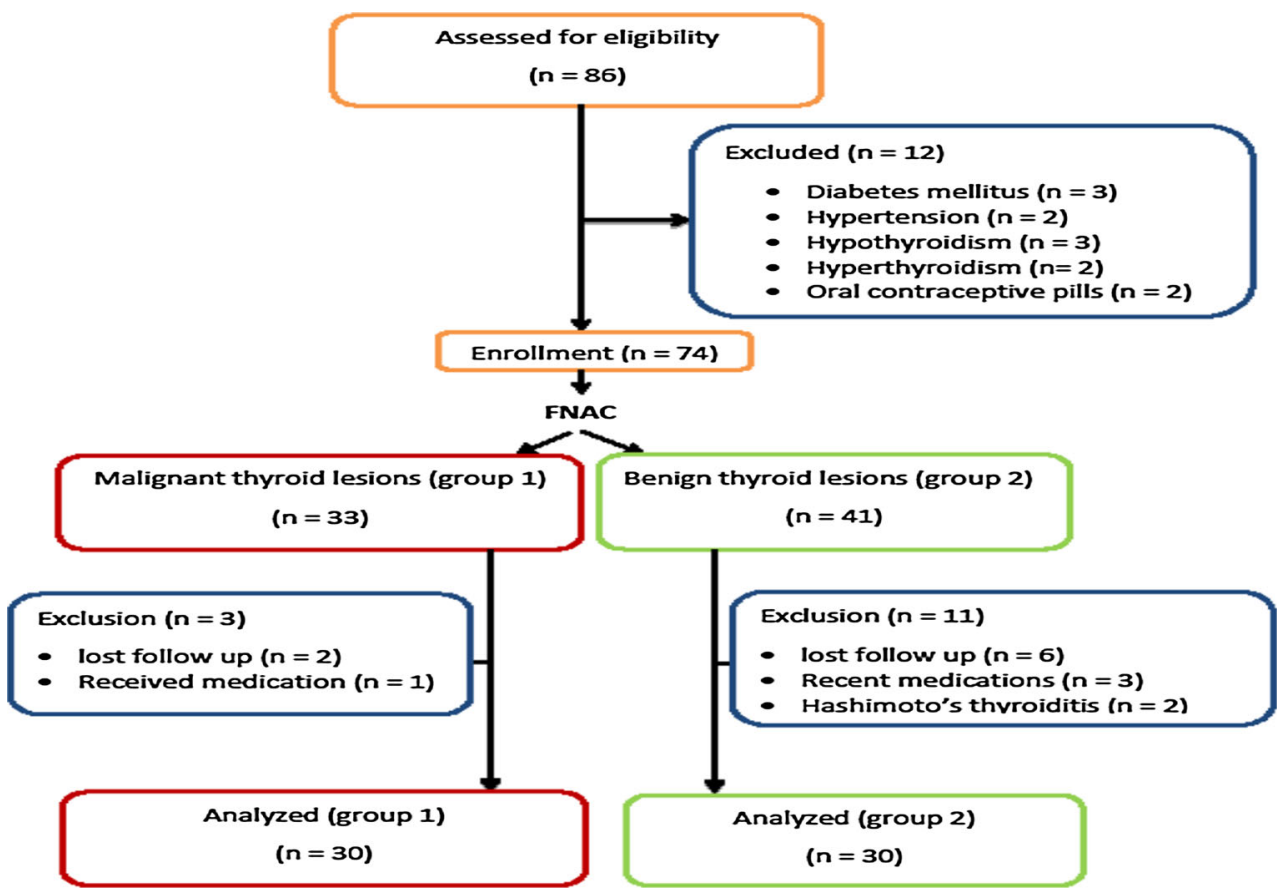

Table 1 Relationship between demographic data and leptin expression

\begin{tabular}{llll}
\hline & Group 1 & Group 2 & $p$ value \\
\hline Gender & & & \\
$\quad$ Male & $12(40.0 \%)$ & $6(20.0 \%)$ & 0.091 \\
Female & $18(60.0 \%)$ & $24(80.0 \%)$ & \\
Age, years & $55.50(18.0-67.0)$ & $35.50(22.0-67.0)$ & 0.157 \\
BMI & $24.87 \pm 5.84$ & $25.03 \pm 4.51$ & 0.902 \\
Menstrual status & $n=18$ & $n=24$ & \\
Pre-menopause & $13(72.2 \%)$ & $20(83.3 \%)$ & 0.462 \\
Post-menopause & $5(27.8 \%)$ & $4(16.7 \%)$ & \\
\hline
\end{tabular}

recorded and compared in both study groups with no significant statistical difference The menstrual status of female patients in both groups was compared with no significance between studied groups $(p=0.462)$ (Table 1$)$.

Comparison between serum leptin levels in both study groups

Both preoperative and postoperative serum leptin levels were measured in the two study groups. The preoperative serum leptin levels were significantly higher in the WDTC patients than in the controls $(19.25[1.50-109.60]$ vs 0.90 $[0.50-11.80] \mathrm{ng} / \mathrm{ml} ; \quad p<0.001$, group 1 vs group 2 , respectively) (Table 2).

There was a significant drop in serum leptin levels 1 month after surgery in the WDTC group, falling from $19.25(1.50-109.60)$ to $0.90(0.60-8.90) \mathrm{ng} / \mathrm{ml}(p<0.001)$.
Table 2 Comparison between cases and controls regarding leptin level

\begin{tabular}{llll}
\hline & Group 1 & Group 2 & $p$ value \\
\hline $\begin{array}{l}\text { Preoperative } \\
\text { leptin level, } \\
\text { ng/ml }\end{array}$ & $19.25(1.50-109.60)$ & $0.90(0.50-11.80)$ & $<0.001^{*}$ \\
$\begin{array}{l}\text { Postoperative } \\
\text { leptin level, } \\
\text { ng/ml }\end{array}$ & $0.90(0.60-8.90)$ & $0.80(0.50-10.80)$ & 0.532 \\
$p$ value & $<0.001 *$ & 0.274 & \\
\hline Statistically significant & &
\end{tabular}

In contrast, for the control group there was no significant difference between preoperative and postoperative serum leptin levels $(p=0.274)$.

One month after surgery, there was no significant difference between serum leptin levels in both groups: 0.90 $(0.60-8.90)$ vs $0.80(0.50-10.80) \mathrm{ng} / \mathrm{ml}$, group 1 vs group 2 respectively $(p=0.532)$.

Comparison between leptin levels in different BMI subgroups

A subanalysis of the two groups was performed based on the patients' BMI. They were subdivided into underweight (BMI $<18$ ), normal (BMI 18 to $<25$ ), overweight (BMI 25 to $<30$ ), and obese (BMI $\geq 30$ ) (Table 3). Leptin levels were measured and compared in the four subgroups before and after surgery. There was no significant difference 
between the two groups regarding BMI. There was, however, a significant postoperative decrease in serum leptin levels in all BMI subgroups in WDTC patients when compared to their preoperative serum leptin levels (Table 4).

Comparison between leptin levels according to menopausal status

Of 30 patients in the WDTC group, $18(60 \%)$ were females, and in the control group there were $24(80 \%)$ females. Female patients in both groups were classified according to their menstrual status into premenopausal or postmenopausal (Table 5). There was no statistically significant difference between preoperative serum leptin levels in premenopausal and postmenopausal WDTC female patients: $11.50(1.50-44.80)$ and $34.90(10.0-34.90) \mathrm{ng} / \mathrm{ml}$, respectively $(p=0.213)$.

There was a significant decrease in serum leptin levels one month postoperatively for both premenopausal and postmenopausal WDTC patients $(p=0.001$ and 0.038$)$, respectively; however, the postmenopausal group of patients still had significantly higher serum leptin levels $8.90(6.90-8.90) \mathrm{ng} / \mathrm{ml}$ in comparison to the premenopausal group $2.10(0.60-6.80) \mathrm{ng} / \mathrm{ml}(p=0.001)$.

In the control group there was no significant difference in serum leptin levels between pre- and postmenopausal female patients either preoperatively or postoperatively ( $p=0.640$ and 0.784), respectively, and there was no significant drop in serum leptin levels after surgery in both pre- and postmenopausal patients groups $(p=0.115$ and $1.0)$.

Table 3 Comparison between both groups regarding BMI

\begin{tabular}{lrrr}
\hline & Group 1 & Group 2 & $p$ value \\
\hline BMI & & & \\
Underweight $(<18)$ & $5(16.7)$ & $1(3.3)$ & 0.195 \\
Normal $(18$ to $<25)$ & $11(36.7)$ & $13(43.3)$ & 0.598 \\
Overweight $(25$ to $<30)$ & $7(23.3)$ & $11(36.7)$ & 0.260 \\
Obese $(\geq 30)$ & $7(23.3)$ & $5(16.7)$ & 0.519 \\
$p$ value & 0.256 & & \\
\hline
\end{tabular}

Relationship between clinicopathological criteria and leptin expression

The postoperative histological examination of specimens from patients with a diagnosis of WDTC showed that $25(83 \%)$ patients had PTC, $2(7 \%)$ patients had FVPTC, and $3(10 \%)$ patients had follicular carcinoma. The comparison of leptin levels of these three groups showed no significant difference $(p=0.106)$, as shown in Fig. 2.

Lymph node metastases were detected in 20 (66.6\%) patients in the WDTC group. There was a trend for preoperative serum leptin levels to be higher in patients with cervical lymph node metastasis-34.25 (1.50-109.60) ng/ ml-compared to those without lymph node metastasis$19.25(4.20-44.80) \mathrm{ng} / \mathrm{ml}$, however did not reach statistical significance $(p=0.480)$. There was also a trend for serum leptin levels to be higher in multifocal primary thyroid tumors $(p=0.064$; Fig. 2$)$. A positive trend was also apparent between the size of the tumor and preoperative leptin level; however, it did not reach a significant value $(p=0.079$; Fig. 3).

Table 5 Comparison between preoperative and postoperative leptin levels in different groups according to menstrual status

\begin{tabular}{llll}
\hline & Pre-menopause & Post-menopause & $p$ value \\
\hline Group 1 & & & \\
$n$ & 13 & 5 & \\
$\begin{array}{c}\text { Preoperative } \\
\text { leptin }\end{array}$ & $11.50(1.50-44.80)$ & $34.90(10.0-34.90)$ & 0.213 \\
$\begin{array}{c}\text { Postoperative } \\
\text { leptin }\end{array}$ & $2.10(0.60-6.80)$ & $8.90(6.90-8.90)$ & $0.001^{*}$ \\
$p$ value & $0.001^{*}$ & $0.038^{*}$ & \\
$\begin{array}{c}\text { Group 2 } \\
n\end{array}$ & 20 & 4 & 0.640 \\
$\begin{array}{l}\text { Preoperative } \\
\text { leptin }\end{array}$ & $2.55(0.50-11.80)$ & $3.55(0.60-7.70)$ & \\
$\begin{array}{c}\text { Postoperative } \\
\text { leptin }\end{array}$ & $0.80(0.50-10.80)$ & $3.20(0.70-6.50)$ & 0.784 \\
$p$ value & 0.115 & 1.000 & \\
\hline
\end{tabular}

* Statistically significant
Table 4 Comparison between preoperative and postoperative leptin levels in WDTC with respect to BMI subgroups

* Statistically significant

\begin{tabular}{lllll}
\hline & $\begin{array}{l}\text { Underweight } \\
(<18)(n=5)\end{array}$ & $\begin{array}{l}\text { Normal }(18 \text { to } \\
<25)(n=11)\end{array}$ & $\begin{array}{l}\text { Overweight }(25 \text { to } \\
<30)(n=7)\end{array}$ & $\begin{array}{l}\text { Obese }(\geq 30) \\
(n=7)\end{array}$ \\
\hline $\begin{array}{c}\text { Preoperative leptin } \\
\text { level, ng/ml }\end{array}$ & $24.0(11.50-24.0)$ & $4.20(1.50-109.60)$ & $22.80(15.70-57.0)$ & $34.90(10.0-44.80)$ \\
$\begin{array}{c}\text { Postoperative leptin } \\
\text { level, ng/ml }\end{array}$ & $0.70(0.70-3.70)$ & $0.80(0.60-2.10)$ & $0.80(0.60-4.50)$ & $6.90(6.80-8.90)$ \\
$p$ value & $0.038^{*}$ & $0.003^{*}$ & $0.017^{*}$ & $0.017^{*}$ \\
\hline
\end{tabular}




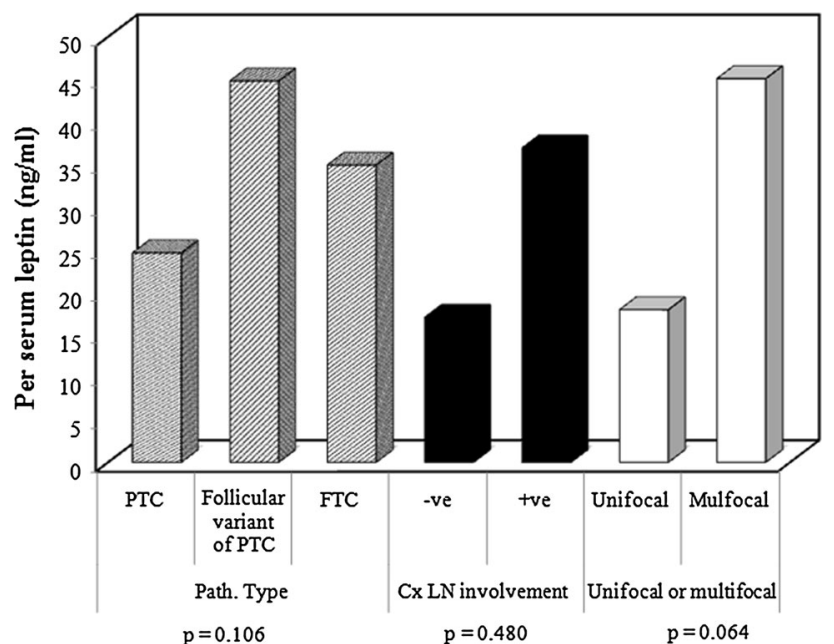

Fig. 2 A correlation between histolopathological characteristics and leptin levels. There was no correlation between serum leptin levels and tumor type $(p=0.106)$, cervical lymph node metastases $(p=0.48)$ or number of foci within the thyroid gland $(p=0.064)$, although trends were observed

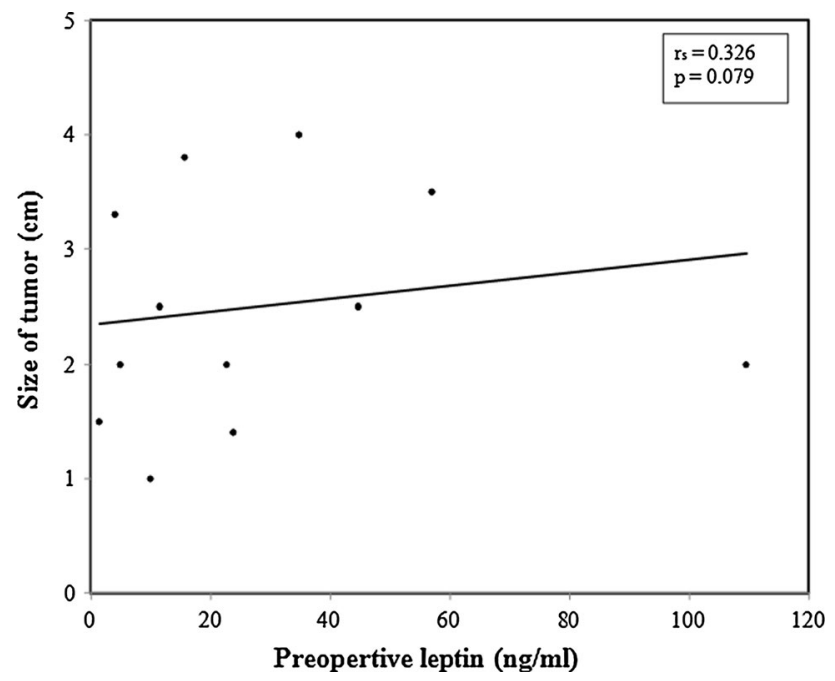

Fig. 3 A correlation between primary tumour size and leptin levels. No correlation was observed between the primary cancer size within the thyroid gland and leptin levels $(p=0.079)$

\section{Discussion}

Several studies have shown that leptin can act as a growth factor promoting proliferation of transformed cells and stimulating angiogenesis in cancers like endometrium and breast $[15,16]$. There is a proven relationship between thyroid gland function and leptin, as both leptin and thyroid hormones regulate the metabolic machinery, as well as energy storage and expenditure. It has been shown that leptin serum levels fluctuate in response to alterations in thyroid activity [17]. This correlation between leptin and thyroid hormone status has been extensively studied, with discrepancies in published results [18-21]. An explanation for these controversial results may arise from the difficulty in matching patients to controls regarding the metabolic status, as the BMI, which is the most commonly used confounding factor is not an accurate reflection of body composition.

Owing to the relation between leptin and both cancer development and the thyroid gland, we hypothesized whether leptin levels would correlate with a diagnosis of thyroid cancer and whether or not higher levels would predict a more aggressive tumor behavior with a greater potential to invade and metastasize. This would reflect on the strictness and duration of patient follow-up and would highlight possibilities of future disease recurrence. This is especially important in Egypt with limited follow-up facilities due to the rural nature, resulting in patients being lost to follow-up and re-presenting several years later with very aggressive disease, as previously reported by our group [22]. In the present study we compared changes in serum leptin levels in patients with WDTC before and after surgery and in control subjects.

Two factors in our study support the relationship between leptin and thyroid cancer. First, we found that the serum leptin levels in WDTC patients were higher than those in their control group counterparts $(p<0.001)$. Second, there was a significant drop in leptin levels 1 month after thyroidectomy compared to pre-thyroidectomy levels. It has been reported that in states of thyroid hormone deficiency, leptin levels increase in parallel with overexpression of thyrotropin releasing hormone and thyroid stimulating hormone (TSH) [17]. It would have therefore been acceptable if leptin levels had increased post-thyroidectomy as a result of the occurrence of intentional hypothyroidism, as we do not replace our thyroid cancer patients with thyroid hormones prior to radioactive iodine ablation. The serum leptin levels did not rise, and the significant decrease in the leptin levels in WDTC patients were paradoxical, and therefore support the concept of a relationship between leptin and thyroid cancer, making leptin a possible etiologic factor in thyroid carcinogenesis. In the benign disease group, there was no significant change in serum leptin levels, possibly due to the fact that these patients were immediately replaced with thyroxine and were euthyroid at the time of leptin measurement.

Following documentation of the primary hypothesis, we sought to investigate other factors that might have influenced leptin serum levels in either group. Because leptin is synthesized and secreted mainly by adipose tissue, and because its plasma levels strongly correlate with BMI [23], we calculated the patients' BMI in both groups before and after surgery; we could not, however, detect any significant 
differences. Moreover, leptin levels decreased postoperatively in all subgroups of WDTC patients irrespective of BMI.

Because leptin may also be affected by menopausal status due to associated hormonal changes, both groups were analyzed based on their menopausal status. Although no differences could be detected in either group preoperatively, both premenopausal and postmenopausal women in the WDTC group had a significant reduction in leptin levels following surgery. The postoperative leptin levels in WDTC postmenopausal women, however, remained significantly higher when compared to premenopausal women. This finding contradicts previous studies that reported a decline in serum leptin levels with advancing age and in postmenopausal women [24, 25]. The lack of difference in leptin levels between premenopausal and postmenopausal women in the WDTC group might have been masked by the presence of the cancer as documented earlier. This would have been expected to drop following surgery, especially in the postmenopausal subgroup. The significantly higher leptin levels in postmenopausal women following thyroid surgery could be explained by the hypothyroid status itself, as this did not occur in the benign disease group patients, who were euthyroid. Teixeira et al. [26] reported that in postmenopausal women, there is a stronger influence of thyroid status on leptin levels through a stronger correlation between TSH and leptin leading to higher leptin levels in this population. They showed that this correlation did not occur in premenopausal women; moreover, leptin levels were reduced with thyroxine replacement.

We then investigated the presence of a correlation between histopathological findings and serum leptin levels. In WDTC, the size of the primary tumor, multifocality, and lymph node status are essential criteria in risk stratification into either low risk or high risk and will predict disease recurrence $[27,28]$. Although trends were demonstrated between leptin levels and each of tumor size $(p=0.079)$, malignant lymph node involvement $(p=0.48)$, and multifocal disease $(p=0.064)$, the differences did not reach statistical significance. Cheng et al. [29, 30] observed a strong association between leptin tissue expression and both tumor size and metastatic lymph nodes in their clinicopathological study, with further work showing the role of leptin in tumor cell migration in PTC.

To conclude, higher leptin levels were associated with a diagnosis of WDTC with a significant drop in the leptin levels following surgery. Other prognostic factors, like lymph node status, age, tumor size, and multifocal disease, had no effect on leptin levels. Future studies should focus on finding a correlation between cancer activation pathways, leptin receptor tissue expression, and serum leptin levels, which could then be used as a tumor marker in the clinical setting.

Open Access This article is distributed under the terms of the Creative Commons Attribution License which permits any use, distribution, and reproduction in any medium, provided the original author(s) and the source are credited.

\section{References}

1. Flegal KM, Carroll MD, Kit BK et al (2012) Prevalence of obesity and trends in the distribution of body mass index among US adults, 1999-2010. JAMA 307:491-497

2. Lang K, Ratke $\mathbf{J}$ (2009) Leptin and adiponectin: new players in the field of tumor cell and leukocyte migration. Cell Commun Signal 7:27

3. Galic S, Oakhill JS, Steinberg GR (2010) Adipose tissue as an endocrine organ. Mol Cell Endocrinol 316:129-139

4. Wu MH, Chou YC, Chou WY et al (2009) Circulating levels of leptin, adiposity and breast cancer risk. Br J Cancer 100:578-582

5. Gonullu G, Kahraman H, Bedir A et al (2010) Association between adiponectin, resistin, insulin resistance, and colorectal tumors. Int J Colorectal Dis 25:205-212

6. Renehan AG, Roberts DL, Dive C (2008) Obesity and cancer: pathophysiological and biological mechanisms. Arch Physiol Biochem 114:71-83

7. Hoda MR, Keely SJ, Bertelsen LS et al (2007) Leptin acts as a mitogenic and antiapoptotic factor for colonic cancer cells. Br J Surg 94:346-354

8. Wauters M, Considine RV, Van Gaal LF (2000) Human leptin: from an adipocyte hormone to an endocrine mediator. Eur $\mathrm{J}$ Endocrinol 143:293-311

9. Garofalo C, Surmacz E (2006) Leptin and cancer. J Cell Physiol 207:12-22

10. Hardie LJ, Guilhot N, Trayhurn P (1996) Regulation of leptin production in cultured mature white adipocytes. Horm Metab Res 28:685-689

11. Podnos YD, Smith DD, Wagman LD et al (2007) Survival in patients with papillary thyroid cancer is not affected by the use of radioactive isotope. J Surg Oncol 96:3-7

12. Chen AY, Jemal A, Ward EM (2009) Increasing incidence of differentiated thyroid cancer in the United States. Cancer 115:3801-3807

13. Sheils $O$ (2005) Molecular classification and biomarker discovery in papillary thyroid carcinoma. Expert Rev Mol Diagn 5:927-946

14. Han JM, Kim TY, Jeon MJ et al (2013) Obesity is a risk factor for thyroid cancer in a large, ultrasonographically screened population. Eur J Endocrinol 168:879-886

15. Mantzos F, Vanakara P, Samara S et al (2011) Leptin receptor expression in neoplastic and normal ovarian and endometrial tissue. Eur J Gynaecol Oncol 32:84-86

16. Macciò A, Madeddu C (2011) Obesity, inflammation, and postmenopausal breast cancer: therapeutic implications. Sci World J 11:2020-2036

17. Botella-Carretero JI, Alvarez-Blasco F, Sancho J et al (2006) Effects of thyroid hormones on serum levels of adipokines as studied in patients with differentiated thyroid carcinoma during thyroxine withdrawal. Thyroid 16:397-402

18. Zimmermann-Belsing T, Brabant G, Holst JJ et al (2003) Circulating leptin and thyroid dysfunction. Eur $\mathrm{J}$ Endocrinol 149:257-271 
19. Santini F, Marsili A, Mammoli C et al (2004) Serum concentrations of adiponectin and leptin in patients with thyroid dysfunctions. J Endocrinol Invest 27:RC5-7

20. Braclik M, Marcisz C, Giebel S et al (2008) Serum leptin and ghrelin levels in premenopausal women with stable body mass index during treatment of thyroid dysfunction. Thyroid 18: 545-550

21. Iglesias P, Alvarez Fidalgo P, Codoceo R et al (2003) Serum concentrations of adipocytokines in patients with hyperthyroidism and hypothyroidism before and after control of thyroid function. Clin Endocrinol 59:621-629

22. Nabawi AS, Al Wagih HF, Hemeida MA et al (2012) Fungating thyroid cancer: a complex clinical scenario. World J Surg 36:598-606. doi:10.1007/s00268-011-1393-z

23. Considine RV, Sinha MK, Heiman ML et al (1996) Serum immunoreactive-leptin concentrations in normal-weight and obese humans. N Engl J Med 34:292-295

24. Ryan AS, Elahi D (1996) The effects of acute hyperglycemia and hyperinsulinemia on plasma leptin levels: its relationships with body fat, visceral adiposity, and age in women. J Clin Endocrinol Metab 81:4433-4438

25. Rosenbaum M, Nicolson M, Hirsch J et al (1996) Effects of gender, body composition, and menopause on plasma concentrations of leptin. J Clin Endocrinol Metab 81:3424-3427
26. Teixeira PF, Cabral MD, Silva NA et al (2009) Serum leptin in overt and subclinical hypothyroidism: effect of levothyroxine treatment and relationship to menopausal status and body composition. Thyroid 19:443-450

27. Randolph GW, Duh QY, Heller KS, American Thyroid Association Surgical Affairs Committee's Taskforce on Thyroid Cancer Nodal et al (2012) The prognostic significance of nodal metastases from papillary thyroid carcinoma can be stratified based on the size and number of metastatic lymph nodes, as well as the presence of extranodal extension. Thyroid 22:1144-1152

28. Roti E, degli Uberti EC, Bondanelli M et al (2008) Thyroid papillary microcarcinoma: a descriptive and meta-analysis study. Eur J Endocrinol 159:659-673

29. Cheng SP, Chi CW, Tzen CY et al (2010) Clinicopathologic significance of leptin and leptin receptor expressions in papillary thyroid carcinoma. Surgery 147:847-853

30. Cheng SP, Yin PH, Hsu YC et al (2011) Leptin enhances migration of human papillary thyroid cancer cells through the PI3K/AKT and MEK/ERK signaling pathways. Oncol Rep 26:1265-1271 